\title{
Directional sensing of deformed cells under faint gradients
}

\author{
Akinori Baba, Tetsuya Hiraiwa, and Tatsuo Shibata \\ Laboratories for Physical Biology, RIKEN Center for Developmental Biology, 2-2-3 Minatojima-minamimachi, \\ Chuo-ku, Kobe 650-0047, Japan and \\ Japan Science and Technology Agency, CREST, 1-3 Yamadaoka, Suita, Osaka 565-0871, Japan
}

(Received 27 July 2012; published 14 December 2012)

\begin{abstract}
We consider the physical limit of the directional sensing ability of living cells, as in chemotaxis, under a low concentration and shallow chemoattractant gradient. Elliptic cells sense the direction, which is a stochastic variable of a characteristic distribution with peaks at directions not necessarily to the gradient. The peak positions depend on the information of the gradient that cells use to infer the direction and also the shape and orientation of cells. Cells of different shapes may use different inference strategies to increase their directional sensing performance.
\end{abstract}

DOI: 10.1103/PhysRevE.86.060901

Living cells are inherently stochastic systems and their surroundings similarly fluctuate. Even under such conditions, cells properly sense and transduce their extracellular and intracellular signals by using stochastic cellular apparatuses. Physical and biochemical mechanisms that underlie these cellular processes are largely unknown [1-4]. Among such cellular processes is eukaryotic chemotaxis, which plays an important role in environmental foraging behavior, morphogenesis, and immune responses. In eukaryotic chemotaxis, chemomolecules stochastically bound to cell receptors enable cells to sense the direction of an extracellular gradient $[5,6]$. Dictyostelium cells show chemotaxis for a few percent of concentration gradient per cell length $(10-30 \mu \mathrm{m})$ in a wide dynamic range from $10 \mathrm{pM}$ to $10 \mu \mathrm{M}$ of local chemoattractant concentration [7-9]. This suggests that the cells can sense the direction of a shallow and low concentration gradient by performing a highly efficient estimation of the direction [10-13]. However, the efficiency of the directional estimation, and the mechanism that underlies such an efficient estimation, are not well understood. To answer these questions, along with understanding a particular molecular mechanism that performs the direction sensing in a particular type of cell, we need to understand the physical limit of accurate directional sensing independent of a particular mechanism, considering the most probable direction of the gradient for a given distribution of chemomolecules bound to the receptors on the cell surface [12-15]. Such an estimation procedure is the maximum likelihood method.

Stochastic fluctuations in the spatial distribution of the bound receptors inevitably introduce stochastic fluctuations in the direction estimated by cells. Previously, in Ref. [14], the estimated direction was considered to be distributed according to the normal distribution, assuming that the number of receptors bound to the chemomolecule is sufficiently large. However, since the distribution of the estimated direction is governed by the directional statistics, it does not necessarily follow that a normal distribution will result in the shallow gradient or low concentration limits.

Published by the American Physical Society under the terms of the Creative Commons Attribution 3.0 License. Further distribution of this work must maintain attribution to the author(s) and the published article's title, journal citation, and DOI.
PACS number(s): 87.16.Xa, 87.10.Mn, 87.17.Jj, 87.18.Tt

Therefore, in this Rapid Communication, based on the idea of maximum likelihood, we study the probability distribution of the estimated chemoattractant gradient by an elliptical cell in a shallow and low concentration gradient. Furthermore, since the outcome of maximum likelihood can depend on the model assumptions, we consider two cases: (1) The cells estimate both the direction and steepness of the gradient, and (2) the cells estimate the direction of the gradient when the steepness is known. We show that differences in the model assumptions qualitatively affect the probability distribution of the estimated direction under shallow gradient or low concentration conditions.

Gradient sensing of an elliptic cell. We consider an elliptic cell with the lengths of major and minor radii given by $a$ and $b(<a)$, respectively (Fig. 1). The cell is exposed to an exponential chemical gradient of steepness $s$, and the long axis is oriented to the angle $-\phi$ from the gradient. The cell perceives the chemoattractant concentration around its periphery, where $N$ receptors are uniformly distributed on the periphery. Thus, the chemoattractant concentration at the $n$th receptor (solid circle in Fig. 1), $\left(r_{n} \cos \varphi_{n}, r_{n} \sin \varphi_{n}\right)$, is $C_{n}=C_{0} \exp \left[s r_{n} \cos \left(\varphi_{n}-\phi\right)\right]$. Here, $r_{n}$ and $\varphi_{n}$ are the distance between the $n$th receptor and the center of the cell and the angle from the long axis to the $n$th receptor, respectively, and $C_{0}$ is the chemoattractant concentration at the center of the cell. The $n$th receptor with the dissociation constant $K_{d}$ can be associated or dissociated with a ligand, designated by $x_{n}=1$ and $x_{n}=0$, respectively. Then, by considering the probabilities of both states of individual receptors, the probability $P(\mathbf{X} \mid s, \phi)$ that the instantaneous state of receptors $\mathbf{X}=\left\{x_{1}, x_{2}, \ldots, x_{N}\right\}$ for given $s$ and $\phi$ is given by

$$
P(\mathbf{X} \mid s, \phi)=\prod_{n=1}^{N}\left(\frac{C_{n}}{C_{n}+K_{d}}\right)^{x_{n}}\left(\frac{K_{d}}{C_{n}+K_{d}}\right)^{1-x_{n}} .
$$

For sufficiently large $N$, the probability density function (PDF) approximates to Gaussian,

$$
P(\mathbf{X} \mid s, \phi) \approx \frac{1}{2 \pi \sigma_{Z_{1}} \sigma_{Z_{2}}} \exp \left(-\frac{\left(Z_{1}-\left\langle Z_{1}\right\rangle\right)^{2}}{2 \sigma_{Z_{1}}^{2}}-\frac{\left(Z_{2}-\left\langle Z_{2}\right\rangle\right)^{2}}{2 \sigma_{Z_{2}}^{2}}\right),
$$

where the stochastic variables $Z_{1}=\sum_{n=1}^{N} x_{n} r_{n} \cos \varphi_{n}$ and $Z_{2}=\sum_{n=1}^{N} x_{n} r_{n} \sin \varphi_{n}$, and $\sigma_{Z_{1}}^{2}$ and $\sigma_{Z_{2}}^{2}$ are their respective 


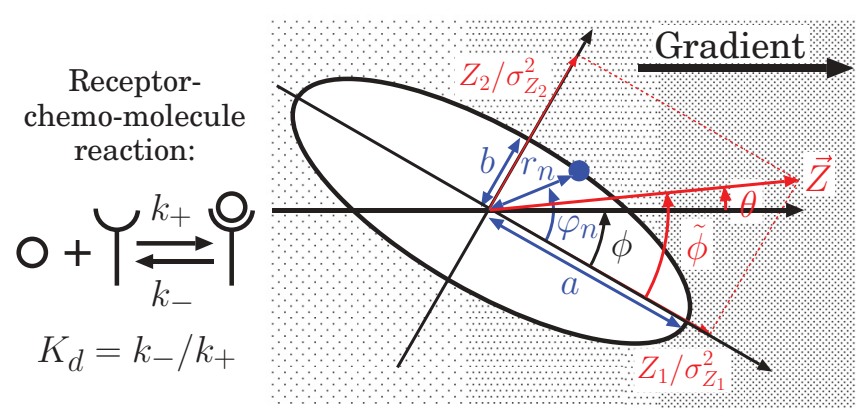

FIG. 1. (Color online) Schematic representation of our model of a eukaryotic chemotactic cell.

variances. The means and variances are calculated from Eq. (1) up to the first order in $s$ as $\left\langle Z_{1}\right\rangle=s \sigma_{Z_{1}}^{2} \cos \phi$, $\left\langle Z_{2}\right\rangle=s \sigma_{Z_{2}}^{2} \sin \phi, \sigma_{Z_{1}}^{2}=\sigma_{0}^{2} \lambda_{1}$, and $\sigma_{Z_{2}}^{2}=\sigma_{0}^{2} \lambda_{2}$. Here, $\sigma_{0}^{2}=$ $N C_{0} K_{d} /\left(C_{0}+K_{d}\right)^{2}$ is the variance of the distribution of the total number of bound receptors if $s=0$, and $\lambda_{1}=$ $N^{-1} \sum_{n=1}^{N} r_{n}^{2} \cos ^{2} \varphi_{n}$ and $\lambda_{2}=N^{-1} \sum_{n=1}^{N} r_{n}^{2} \sin ^{2} \varphi_{n}$ characterize the cell shape [16].

Estimating both direction and steepness. As stated above, we assume that cells use the maximum likelihood to estimate the chemoattractant gradient for a given state of receptors, $\mathbf{X}$. When the cell estimates both direction $\phi$ and gradient steepness $s$, the estimates of $\tilde{s}(\mathbf{X})$ and $\tilde{\phi}(\mathbf{X})$ that maximize the likelihood $\mathbf{L}(\tilde{s}, \tilde{\phi} \mid \mathbf{X}) \equiv P(\mathbf{X} \mid \tilde{s}, \tilde{\phi})$ for a given $\mathbf{X}$ are calculated from Eq. (2) as [14]

$$
\{\tilde{s}(\mathbf{X}) \cos [\tilde{\phi}(\mathbf{X})], \tilde{s}(\mathbf{X}) \sin [\tilde{\phi}(\mathbf{X})]\}=\left(\frac{Z_{1}}{\sigma_{Z_{1}}^{2}}, \frac{Z_{2}}{\sigma_{Z_{2}}^{2}}\right) .
$$

Thus, the estimated direction of the gradient is the argument of the vector $\vec{Z}=\left(Z_{1} / \sigma_{Z_{1}}^{2}, Z_{2} / \sigma_{Z_{2}}^{2}\right)$ given by the right-hand side of Eq. (3) (Fig. 1). Here, $Z_{1} / \sigma_{Z_{1}}^{2}$ and $Z_{2} / \sigma_{Z_{2}}^{2}$ are considered roughly proportional to the difference in the number of bound receptors between the ends along the long axis of the cell, and between the sides along the short axis, respectively. The respective averages, given by $(s \cos \phi, s \sin \phi)$, are independent of $N$ and $C_{0}$. However, the distributions of the estimates of gradient directions depend on $N$ and $C_{0}$.

Because $\mathbf{X}$ is a random variable distributed according to Eq. (1), the estimated values $\tilde{s}$ and $\tilde{\phi}$ are also considered random variables. By summing the probability of $\mathbf{X}$ that yields a particular $\tilde{s}$ and $\tilde{\phi}$, the PDF of $\tilde{s}$ and $\tilde{\phi}$ is written as

$$
\mathcal{P}(\tilde{s}, \tilde{\phi} \mid s, \phi)=\int d \mathbf{X} P(\mathbf{X} \mid s, \phi) \delta(\tilde{s}-\tilde{s}(\mathbf{X})) \delta(\tilde{\phi}-\tilde{\phi}(\mathbf{X})) .
$$

Because the gradient direction (rather than the steepness $s$ ) supplies the information necessary for cells to determine their next direction to move, we consider the $\operatorname{PDF} \mathcal{P}(\tilde{\phi})$ of the estimated direction by integrating $\mathcal{P}(\tilde{s}, \tilde{\phi})$ with respect to $\tilde{s}$. Hereafter, we consider the angle $\theta=\tilde{\phi}-\phi$ between the estimated direction and the chemoattractant gradient direction (Fig. 1). The $\operatorname{PDF} \mathcal{P}(\theta)$ is then given by

$$
\begin{aligned}
\mathcal{P}(\theta) & =\int_{0}^{\infty} \mathcal{P}(\tilde{s}, \tilde{\phi}=\theta+\phi \mid s, \phi) d \tilde{s} \\
& =\frac{\sqrt{\lambda_{1} \lambda_{2}}}{2 \pi} \mathcal{Q}(\theta) \cdot \mathcal{R} \cdot \mathcal{S}(\theta),
\end{aligned}
$$

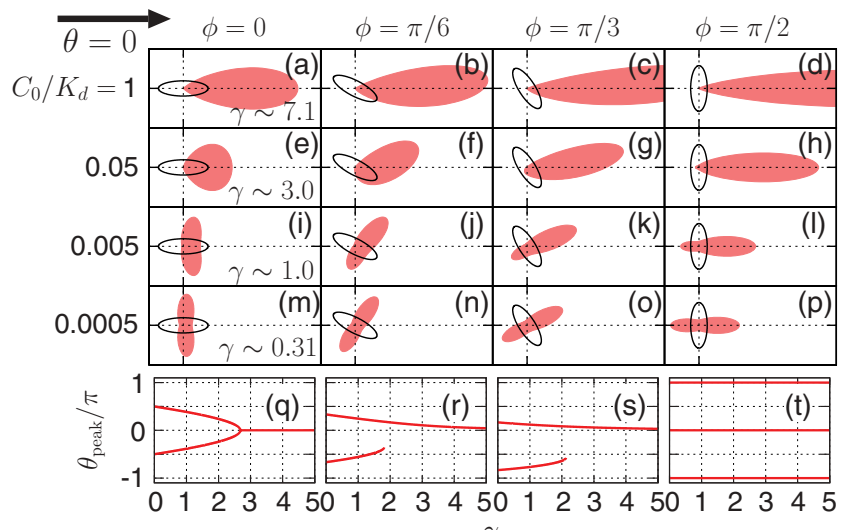

FIG. 2. (Color online) Distribution of the estimated direction of chemoattractant gradient. (a)-(p) $\mathcal{P}(\theta)$ for various concentrations and cell orientations. The chemoattractant gradient direction, $\theta=0$, points to the right in the figure, and $\mathcal{P}(\theta)$ is represented as the distance from the center (the intersection of the two dotted lines) to the periphery of the red (light gray) region at angle $\theta$. From top to bottom: $\gamma \sim 7.1,3.0,1.0$, and $0.31\left(C_{0} / K_{d}=1,0.05,0.005\right.$, and $0.0005)$. From left to right: $\phi=0, \pi / 6, \pi / 3$, and $\pi / 2$. The open black ellipses indicate cell shape and orientation. (q)-(t) The peak angles $\theta_{\text {peak }}$ for which $\mathcal{P}(\theta)$ is maximal, plotted as functions of $\gamma$. From left to right: $\phi=0, \pi / 6, \pi / 3$, and $\pi / 2$. Other parameter values are $s=0.05, N=80000, r_{0}=1$ (where 1 distance unit $=10 \mu \mathrm{m}$ ), and $a / b=3(\epsilon \sim 0.94)$.

where $\gamma=s \sigma_{0}$,

$$
\begin{aligned}
\mathcal{Q}(\theta)= & \frac{1}{\lambda_{1} \cos ^{2}(\theta+\phi)+\lambda_{2} \sin ^{2}(\theta+\phi)}, \\
\mathcal{R}= & \exp \left(-\frac{1}{2} \gamma^{2}\left(\lambda_{1} \cos ^{2} \phi+\lambda_{2} \sin ^{2} \phi\right)\right), \\
\mathcal{S}(\theta)= & 1+\gamma H(\theta) \sqrt{\frac{\pi}{2}} \operatorname{erfc}\left(-\frac{1}{\sqrt{2}} \gamma H(\theta)\right) \\
& \times \exp \left(\frac{1}{2} \gamma^{2} H(\theta)^{2}\right),
\end{aligned}
$$

with

$$
H(\theta)=\frac{\lambda_{1} \cos (\theta+\phi) \cos \phi+\lambda_{2} \sin (\theta+\phi) \sin \phi}{\sqrt{\lambda_{1} \cos ^{2}(\theta+\phi)+\lambda_{2} \sin ^{2}(\theta+\phi)}},
$$

and $\operatorname{erfc}(x)=1-(2 / \sqrt{\pi}) \int_{0}^{x} \exp \left(-t^{2}\right) d t$.

In Fig. 2, $\mathcal{P}(\theta)$ is shown for different values of $\gamma$ and cell orientations. Here, we select parameter values that typify Dictyostelium cells. For sufficiently large values of $\gamma$ [Figs. 2(a)-2(d)], the estimated directions are distributed around a peak close to $\theta=0$, irrespective of the cell orientations, as is expected from the maximum likelihood. As $\gamma$ decreases, however, the distribution increasingly deviates from a Gaussian-like unimodal distribution, eventually forming two peaks in the short axial direction. At sufficiently small $\gamma$, the peaks in the distribution of the estimated directions are found solely along the short axis [Figs. 2(m)-2(p)]. Thus, when a cell is oriented towards a chemoattractant gradient under these conditions, the estimated direction tends perpendicularly to the true gradient [Fig. 2(m)]. 
To understand whether and how the distribution of the estimated gradient qualitatively depends on $\gamma$, we next study the dependence on $\gamma$ of the angles $\theta_{\text {peak }}$ that give the local maxima of $\mathcal{P}(\theta)$, as shown in Figs. 2(q)-2(t). For small $\gamma$ and small deformation $\epsilon^{2}=1-(b / a)^{2}$, the first-order approximation to $\mathcal{P}(\theta)$ is

$$
\begin{aligned}
\mathcal{P}(\theta)= & \frac{1}{2 \pi}\left[1+\frac{\sqrt{\pi}}{2} r_{0} \gamma \cos \theta-\frac{3 \epsilon^{2}}{8} \cos 2(\theta+\phi)\right] \\
& +o\left(r_{0} \gamma\right)+o\left(\epsilon^{2}\right),
\end{aligned}
$$

where $r_{0}(=\sqrt{a b})$ is the geometric mean radius. The second and the third terms on the right-hand side of Eq. (6) come from $\mathcal{Q}(\theta)$ and $\mathcal{S}(\theta)$ in Eq. (5), respectively, indicating that $\mathcal{S}(\theta)$ contributes to the peak at the correct estimation $\theta=0$, while $\mathcal{Q}(\theta)$ peaks in the direction of the short axis. From Eq. (6), it is seen that, for $r_{0} \gamma \gg \epsilon^{2}$, (regardless of $\phi$ ) the contribution from the gradient $\mathcal{S}(\theta)$ dominates, and a single peak emerges in $\mathcal{P}(\theta)$. When $r_{0} \gamma \ll \epsilon^{2}$, the deformation contribution $\mathcal{Q}(\theta)$ dominates, and a bimodal gradient-estimate distribution results.

According to Eq. (3), when $Z_{1} / \sigma_{Z_{1}}^{2}>Z_{2} / \sigma_{Z_{2}}^{2}$, the estimated direction tends towards the long axis, whereas it tends towards the short axis when $Z_{1} / \sigma_{Z_{1}}^{2}<Z_{2} / \sigma_{Z_{2}}^{2}$. Because $\sigma_{Z_{1}}^{2}>\sigma_{Z_{2}}^{2}$, the variance of $Z_{2} / \sigma_{Z_{2}}^{2}$, given by $\left(\sigma_{Z_{2}}\right)^{-2}$, is larger than the variance of $Z_{1} / \sigma_{Z_{1}}^{2}$, given by $\left(\sigma_{Z_{1}}\right)^{-2}$. Thus, $\vec{Z}=$ $\left(Z_{1} / \sigma_{Z_{1}}^{2}, Z_{2} / \sigma_{Z_{2}}^{2}\right)$ fluctuates larger along the short axis than along the long axis, and the probability that $Z_{1} / \sigma_{Z_{1}}^{2}<Z_{2} / \sigma_{Z_{2}}^{2}$ is high (relative to the reverse case) for sufficiently small $\gamma=s \sigma_{0}$. Therefore, for a shallow and low concentration gradient, the estimated direction tends to point around the short axis in this case.

Estimating only direction. We consider another scenario that the cell estimates only the direction of the gradient by a most efficient method, given that the correct steepness $s$ around the cell is known by some other way. As cells move, the change in the steepness around the cell may be much slower than the change in the direction of the cell motion. In Dictyostelium cells, the cellular orientation changes over a time scale from seconds to a few minutes [17], whereas the characteristic time in which steepness changes is tens of minutes (here, we consider that a cell moves in a $2 \%$ in $10 \mu \mathrm{m}$ linear gradient with a cell velocity $v \sim 20 \mu \mathrm{m} / \mathrm{min})$. Therefore, cells may frequently probe the chemoattractant direction while retaining a memory of the gradient magnitude, which is thus updated less frequently.

Here, we use the maximum likelihood to determine the direction $\tilde{\phi}(\mathbf{X})$ that maximizes the likelihood $\mathbf{L}(\tilde{\phi} \mid \mathbf{X}) \equiv$ $P(\mathbf{X} \mid s, \tilde{\phi})$ for a given $\mathbf{X}$ under the condition that $s$ is given. The estimate is the solution of the following equation:

$\lambda_{1} s \sin \tilde{\phi}\left(s \cos \tilde{\phi}-\frac{Z_{1}}{\sigma_{Z_{1}}^{2}}\right)-\lambda_{2} s \cos \tilde{\phi}\left(s \sin \tilde{\phi}-\frac{Z_{2}}{\sigma_{Z_{2}}^{2}}\right)=0$.

Thus, the estimated direction is not necessarily the argument of vector $\vec{Z}$. Consider the estimation vector $\overrightarrow{\mathcal{E}}=(s \cos \tilde{\phi}, s \sin \tilde{\phi})$. In terms of Eq. (7), $\mathcal{E}$ can be written as

$$
\overrightarrow{\mathcal{E}}=\left(\begin{array}{cc}
\frac{\lambda_{1}}{\lambda_{1}-c} & 0 \\
0 & \frac{\lambda_{2}}{\lambda_{2}-c}
\end{array}\right) \vec{Z}
$$

where $c$ is a parameter depending on $\vec{Z}$. For a circular cell, $\lambda_{1}=$ $\lambda_{2}$ and $\overrightarrow{\mathcal{E}}=\vec{Z}$. For a cell of elliptic shape with a small $c \sim 0$, the estimate is close to $\vec{Z}$. The parameter $c$ is approximately 0 , when both $N$ and $C_{0}$ are large values. In this case, both $\vec{Z}$ and $\overrightarrow{\mathcal{E}}$ approach to the vector $(s \cos \phi, s \sin \phi)$. When $|\vec{Z}|$ deviates largely from $|\overrightarrow{\mathcal{E}}|=s, c$ may differ significantly from 0 .

The PDF of $\tilde{\phi}$,

$$
\mathcal{P}_{s}(\tilde{\phi} \mid s, \phi)=\int d \mathbf{X} P(\mathbf{X} \mid s, \phi) \delta(\tilde{\phi}-\tilde{\phi}(\mathbf{X})),
$$

is obtained by integrating along the line given by Eq. (7) in the space of $Z_{1}$ and $Z_{2}$. The PDF of $\theta=\tilde{\phi}-\phi$ is then determined as

$$
\mathcal{P}_{s}(\theta)=\frac{\sqrt{\lambda_{1} \lambda_{2}}}{2 \pi} \mathcal{Q}_{s}(\theta) \mathcal{R}_{s}(\theta) \mathcal{S}_{s}(\theta)
$$

where

$$
\begin{aligned}
& \mathcal{Q}_{s}(\theta)=\frac{1}{\lambda_{2} \cos ^{2}(\theta+\phi)+\lambda_{1} \sin ^{2}(\theta+\phi)}, \\
& \mathcal{R}_{s}(\theta)=\exp \left\{-\frac{1}{2} \gamma^{2}\left[\lambda_{1}\left(\frac{\lambda_{1}-\lambda_{2}}{\lambda_{1}} \cos (\theta+\phi)-\cos \phi\right)^{2}+\lambda_{2} \sin ^{2} \phi\right]\right\} \\
& \mathcal{S}_{s}(\theta)=1+\gamma \sqrt{\frac{\pi}{2}}\left(H_{s}(\theta)+\left(\lambda_{1}-\lambda_{2}\right) \sqrt{\frac{\cos ^{2}(\theta+\phi)}{\lambda_{1}}+\frac{\sin ^{2}(\theta+\phi)}{\lambda_{2}}} \sin ^{2}(\theta+\phi)\right) \operatorname{erfc}\left(-\frac{1}{\sqrt{2}} \gamma H_{s}(\theta)\right) \exp \left(\frac{1}{2} \gamma^{2} H_{s}(\theta)^{2}\right),
\end{aligned}
$$

with

$$
H_{S}(\theta)=\sqrt{\frac{\lambda_{2}}{\lambda_{1}}} \frac{\lambda_{1} \cos \theta-\left(\lambda_{1}-\lambda_{2}\right) \cos ^{2}(\theta+\phi)}{\sqrt{\lambda_{2} \cos ^{2}(\theta+\phi)+\lambda_{1} \sin ^{2}(\theta+\phi)}} .
$$

We note that the distribution of the estimated direction changes smoothly with the presupposed steepness $s$ around its correct value. Thus, the results are mostly insensitive to the presupposed gradient steepness $s$.

In Fig. 3, $\mathcal{P}_{s}(\theta)$ for various concentrations and cell orientations are shown as blue (dark gray) regions. When $\gamma$ is sufficiently large [Figs. 3(a)-3(d)], the estimated direction $\tilde{\phi}$ is distributed around the direction of the true gradient irrespective of the cell's orientation, as in the previous case. As $\gamma$ decreases, the distribution of the estimated directions deviates from 


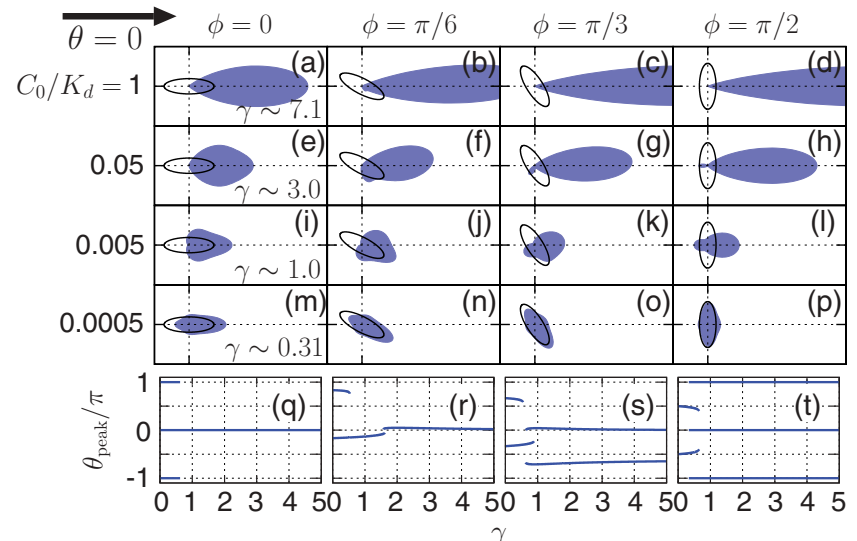

FIG. 3. (Color online) Distribution of the estimated direction when the steepness is known. (a)-(p) $\mathcal{P}_{s}(\theta)$ for various concentration and cell orientations shown as the blue (dark gray) region. (q)-(t) The peak angles $\theta_{\text {peak }}$ plotted as functions of $\gamma$. See Fig. 2 for details.

the Gaussian-like unimodal distribution and becomes more complicated. In the range of intermediate $\gamma$ [Figs. 3(e)-3(1)], with $\phi=\pi / 6, \pi / 3$, and $\pi / 2$, the distributions not only peak at the true gradient direction $\theta=0$, i.e., $\tilde{\phi}=\phi$, but also display an additional small peak at the direction $\theta=-2 \phi$, that is $\tilde{\phi}=$ $-\phi$ the other side of the gradient direction $\phi$ reflected at the direction of the long axis. At low chemoattractant concentrations $\left(r_{0} \gamma \ll 1\right)$, in Figs. 3(m)-3(p), the estimates are distributed around the long axis, in strong contrast to the previous case.

In Figs. 3(q)-3(t), the angles $\theta_{\text {peak }}$ of the peaks in the distribution of Eq. (10) are plotted as functions of $\gamma$. The first-order approximation to $\mathcal{P}_{s}(\theta)$ of Eq. (10) with respect to $r_{0} \gamma$ and $\epsilon^{2}$ is given by

$$
\begin{aligned}
\mathcal{P}_{s}(\theta)= & \frac{1}{2 \pi}\left(1+\frac{\sqrt{\pi}}{2} r_{0} \gamma \cos \theta+\frac{3 \epsilon^{2}}{8} \cos 2(\theta+\phi)\right) \\
& +o\left(r_{0} \gamma\right)+o\left(\epsilon^{2}\right) .
\end{aligned}
$$

By shifting the distribution in Eq. (11) by $\pi / 2$, the distribution of the previous case, Eq. (6), is recovered, indicating that the most probable estimate of the gradient direction differs by $\pi / 2$ between the two cases when $\gamma$ is small. To the first order, Eq. (11) cannot elucidate the peak at the above-mentioned direction $\theta=-2 \phi$ in the other side of the gradient direction, since $\mathcal{R}_{s}(\theta)$ in Eq. (10) is unity at orders lower than $O\left(\gamma^{2} r_{0}^{2} \epsilon^{2}\right)$. We note that the term $\mathcal{R}_{s}(\theta)$ in Eq. (10) at the elliptical limit $\left(\epsilon^{2} \sim 1\right)$ is maximized at both $\theta=0$ and at the angle $\theta=-2 \phi$ [Figs. 3(s)-3(t)].

From Eq. (8), when $|\vec{Z}|>s, c$ is negative so that $\vec{Z}$ is scaled to fit $\overrightarrow{\mathcal{E}}$. Because $\lambda_{1}>\lambda_{2}$, the component of $\vec{Z}$ along the short axis reduces more than the component along the long axis. Consequently, the estimated directions are biased in the direction of the long axis. When $\gamma$ is small, the frequency of $|\vec{Z}|>s$ is larger than that of $|\vec{Z}|<s$. (For instance, consider the case when $s$ is almost zero.) From Eq. (8), as the decrease of $\gamma$ with $-c \gg \lambda_{1}$, the variance of $\overrightarrow{\mathcal{E}}$ is proportional to $\lambda_{1}$ and $\lambda_{2}$, along the long and short axes, respectively. This demonstrates that the fluctuation along the long axis becomes larger than the short axis, and the distribution of the estimated direction becomes biased towards the long axis. On the contrary, when $|\vec{Z}|<s$, $c$ is positive $\left(c<\lambda_{1}, \lambda_{2}\right)$, and $\vec{Z}$ tends towards the short axis.

For smaller $\gamma$, the information along the long axis weights the cell's estimates predominantly in the elongated direction. As $\gamma$ increases, the probability that $|\vec{Z}|<s$ increases, giving rise to the peak at the direction around $\theta=-2 \phi$ in the other side of the gradient direction.

In this Rapid Communication, we investigated the physical limit of the gradient sensing ability of chemotactic cells with an elliptic shape by considering the most probable direction [12-14], for the case that a cell infers both the steepness and direction of the gradient (Fig. 2) and for the case that a cell infers only the direction (Fig. 3). We found that for a faint gradient $(\gamma \ll 1)$ with a low concentration and/or a shallow gradient, a former type of cell prefers the short axial direction, while a latter type of cell prefers the long axial direction. We also found that a latter case also prefers to $\theta=-2 \phi$ at an intermediate gradient $(\gamma \sim 1)$.

A most pertinent question is how cells can perform directional sensing in a shallow chemical gradient. An elongated cell shape may facilitate the gradient sensing ability. Our results demonstrate that the noise present at the association and dissociation reactions of chemomolecules strongly influences the inference of gradient direction. In particular, the elliptic cell shape modulates the error in the estimation in a way that depends on the cell shape and orientation.

Our result could suggest that for efficient chemotaxis the cell deformation and locomotive properties and the direction sensing ability are interdependent on each other. From our result when both steepness and direction are inferred, cells tend to move in the short axial direction. For a chemotactic cell which moves perpendicularly to the long axis as a fish keratocyte, this property may be profitable because a cell may continuously move to the gradient when the short axis is directed to the gradient. On the contrary, when only the direction is inferred, the cells prefer their elongated direction. This tendency is beneficial for the Dictyostelium cells and other types of mammalian chemotactic cells, which typically elongate their bodies to the migration direction.

Based on these considerations, we may be able to elucidate the process of gradient sensing in a particular cell type from experiments by studying the statistics of chemotactic migration direction and cell morphology. To this end, we need to study a motility processes, in which the cell deformation, internal polarity, and direction inference are coupled, and obtain the statistical properties of chemotactic migration and cell morphology [18]. Based on the present study, the distribution of the migration direction has been obtained [18], which shows a good agreement with the experimental data in Ref. [19], suggesting that Dictyostelium cells seem to infer only the gradient direction.

In this way, the most efficient inference processing in a shallow and low concentration gradient can be different for different cell types. In either case, the inference can be improved by temporal integration of the binding information [20,21], though the time interval necessary for accurate 
estimation depends on both the steepness and concentration of the gradient. Directional sensing may also be influenced by the way in which the cell changes its orientation. The physical limits shown in this Rapid Communication have highlighted the need to study further the mechanisms of gradient sensing.

This work was supported by KAKENHI (23111531).
[1] H. C. Berg and E. M. Purcell, Biophys. J. 20, 193 (1977).

[2] T. Shibata and K. Fujimoto, Proc. Natl. Acad. Sci. USA 102, 331 (2005).

[3] W. Bialek and S. Setayeshgar, Proc. Natl. Acad. Sci. USA 102, 10040 (2005).

[4] S. Tănase-Nicola, P. B. Warren, and P. R. ten Wolde, Phys. Rev. Lett. 97, 068102 (2006).

[5] M. Ueda and T. Shibata, Biophys. J. 93, 11 (2007).

[6] P. J. van Haastert and M. Postma, Biophys. J. 93, 1787 (2007).

[7] P. R. Fisher, R. Merkl, and G. Gerisch, J. Cell Biol. 108, 973 (1989).

[8] L. Song et al., Eur. J. Cell Biol. 85, 981 (2006).

[9] D. Fuller et al., Proc. Natl. Acad. Sci. USA 107, 9656 (2010).

[10] W.-J. Rappel and H. Levine, Proc. Natl. Acad. Sci. USA 105, 19270 (2008).

[11] W.-J. Rappel and H. Levine, Phys. Rev. Lett. 100, 228101 (2008).
[12] R. G. Endres and N. Wingreen, Proc. Natl. Acad. Sci. USA 105, 15749 (2008).

[13] B. Hu, W. Chen, W. J. Rappel, and H. Levine, Phys. Rev. Lett. 105, 048104 (2010).

[14] B. Hu, W. Chen, W. J. Rappel, and H. Levine, Phys. Rev. E 83, 021917 (2011).

[15] B. W. Andrews and P. A. Iglesias, PLoS Comput. Biol. 3, e153 (2007).

[16] For $\quad N \gg 1, \quad \lambda_{1} / a^{2} \approx\left(2 \epsilon^{2}-1\right) /\left(3 \epsilon^{2}\right)+\left[\left(1-\epsilon^{2}\right) K\left(\epsilon^{2}\right)\right] /$ $\left[3 \epsilon^{2} E\left(\epsilon^{2}\right)\right]$, and $\lambda_{2} / b^{2} \approx 1-\lambda_{1} / a^{2}$, where $\epsilon=\sqrt{1-(b / a)^{2}}$, $K\left(\epsilon^{2}\right)=\int_{0}^{\pi / 2}\left(1-\epsilon^{2} \sin ^{2} \phi\right)^{-1 / 2} d \phi, \quad$ and $\quad E\left(\epsilon^{2}\right)=$ $\int_{0}^{\pi / 2} \sqrt{1-\epsilon^{2} \sin ^{2} \phi} d \phi$.

[17] H. Takagi et al., PLoS One 3, e2648 (2008).

[18] T. Hiraiwa, A. Baba, and T. Shibata (unpublished).

[19] B. Hu, D. Fuller, W. F. Loomis, H. Levine, and W.-J. Rappel, Phys. Rev. E 81, 031906 (2010).

[20] T. J. Kobayashi, Phys. Rev. Lett. 104, 228104 (2010).

[21] T. J. Kobayashi, Phys. Rev. Lett. 106, 228101 (2011). 